\title{
On the emergence of the $\Lambda \mathrm{CDM}$ model from self-interacting Brans-Dicke theory in $d=5$
}

\author{
Luz Marina Reyes ${ }^{1, a}$, Santiago Esteban Perez Bergliaffa ${ }^{2, b}$ \\ ${ }^{1}$ Departamento de Ciencias Computacionales, CUCEI, Universidad de Guadalajara, Av. Revolución 1500, 44430 Guadalajara, Jal., Mexico \\ 2 Departamento de Física Teórica, Instituto de Física, Universidade do Estado do Rio de Janeiro, Rua São Francisco Xavier 524, Maracanã, Rio de \\ Janeiro, Brazil
}

Received: 13 September 2017 / Accepted: 21 December 2017 / Published online: 11 January 2018

(C) The Author(s) 2018. This article is an open access publication

\begin{abstract}
We investigate whether a self-interacting BransDicke theory in $d=5$ without matter and with a timedependent metric can describe, after dimensional reduction to $d=4$, the FLRW model with accelerated expansion and nonrelativistic matter. By rewriting the effective 4-dimensional theory as an autonomous 3-dimensional dynamical system and studying its critical points, we show that the $\Lambda \mathrm{CDM}$ cosmology cannot emerge from such a model. This result suggests that a richer structure in $d=5$ may be needed to obtain the accelerated expansion as well as the matter content of the 4-dimensional universe.
\end{abstract}

\section{Introduction}

Several observations (such as SNe Ia, baryon acoustic oscillations, and the cosmic microwave background, see for instance [1]) indicate that the universe is currently undergoing an accelerated expansion. In the framework of the Standard Cosmological Model, such an expansion is only possible if matter with unusual properties is added as a source of Einstein's Equations (EEs) [2]. The simplest candidate is the cosmological constant, but there is a huge discrepancy between its theoretical value and the one that follows from observations [3]. Models with scalar or vector fields (generically known as dark energy, see [4] for a review of these and other candidates) have also been considered as the source of the accelerated expansion. Since none of these proposals is free of problems, several alternatives that avoid the introduction of dark energy have been investigated. Among them we can mention theories of gravity that go beyond General Relativity [5] and inhomogeneous cosmological models [6]. Yet another interesting proposal is based on the hypothesis that

\footnotetext{
a e-mail: luzmarinareyes@gmail.com

be-mail: sepbergliaffa@gmail.com
}

the dimensionality of the universe is actually greater than four. The common theme in the many realizations of this idea is that an effective energy-momentum tensor of purely geometrical origin, generated by the reduction of some theory of gravitation defined in $d>4$ to $d=4$, is used to describe the accelerated expansion and/or ordinary matter.

In particular, the reduction of gravitational theories from $d=5$ to $d=4$ has been repeatedly explored in the literature [7]. An appealing example of this type was presented in [8], where the energy-momentum of ordinary matter in $d=4$ arises from the extra-dimensional sector of the theory defined by $G_{A B}=0 .{ }^{1}$ More generally, theories in which the matter content in $d=4$ is induced by dimensional reduction of the vacuum equations of a gravitational theory defined in $d=5$ are known today as induced matter theories (IMTs) [7]. Among many examples of IMTs that have been studied we can mention Brans-Dicke (BD) theory [9-14], ${ }^{2} f(R)$ theories [17,18], and $f(R, T)$ theories [19]. Here we shall investigate the possibility of describing the accelerated expansion of the 4-dimensional universe as well as ordinary pressure-less matter starting from BD theory in the presence of a potential in $d=5 .^{3}$

We shall begin by showing in Sect. 2 that, in an appropriate cosmological setting, the $d=5$ self-interacting BD theory is equivalent to a self-interacting $\mathrm{BD}$ theory in $d=4$ plus an extra scalar field (associated to the time-dependent metric coefficient of the fifth dimension). In Sect. 3, we write the $d=4$ field equations as an autonomous three-dimensional dynamical system, and obtain its critical points, under the assumption that they are deSitter-like (namely, requiring that

\footnotetext{
${ }^{1}$ Latin capital indices $A, B \ldots$ go from 0 to 4 , Greek indices go from 0 to 3 , and latin indices, from 1 to 3 .

2 For $d=5$ BD theory with matter see $[15,16]$.

3 The cosmological evolution in self-interacting BD theory has been examined both in $d=4$ (see for instance [20-22]) and in $d=5$ [23].
} 
$\dot{H}=0)$. We pay special attention to dependence of the eigenvalues of the linearization matrix associated to each critical point with the BD parameter $\omega$, and search for ranges of the latter such that a given critical point is a stable one. In the case such an interval exists, we check whether the system at the critical point can describe the accelerated expansion of the 4-dimensional universe, as well as the pressure-less matter contained in it. We close with some comments in Sect. 4.

\section{Brans-Dicke theory in $d=5$ and its reduction to $\mathrm{d}=4$}

Our starting point is BD theory of gravity in five dimensions, with the action in the Jordan frame given by

$$
\begin{aligned}
{ }^{(5)} \mathcal{S}= & \frac{1}{2 \kappa_{5}} \int d^{5} y \sqrt{{ }^{(5)} \gamma}\left[\phi^{(5)} R-\frac{\omega}{\phi} \gamma^{A B} \nabla_{A} \phi \nabla_{B} \phi\right. \\
& -2 V(\phi)],
\end{aligned}
$$

where ${ }^{(5)} \gamma$, is the determinant of the 5-dimensional metric $\gamma_{A B}, \phi$ is the BD scalar field directly coupled to the 5dimensional Ricci scalar ${ }^{(5)} R, \nabla_{A}$ is the covariant derivative in $d=5, \omega$ is the BD parameter and $V(\phi)$ is the scalar field potential. The variation of the action with respect to $\gamma_{A B}$ yields

$$
\begin{aligned}
{ }^{(5)} G_{A B}= & \kappa_{5}{ }^{(5)} T_{A B}+\frac{\omega}{\phi^{2}}\left[\nabla_{A} \phi \nabla_{B} \phi-\frac{\gamma_{A B}}{2} \nabla^{C} \phi \nabla_{C} \phi\right] \\
& +\frac{1}{\phi}\left[\nabla_{A} \nabla_{B} \phi-\gamma_{A B}{ }^{(5)} \square \phi\right]-\frac{V(\phi)}{\phi} \gamma_{A B},
\end{aligned}
$$

where ${ }^{(5)} \square=\nabla^{A} \nabla_{A}$, and ${ }^{(5)} G_{A B}$ is the Einstein tensor in $d=5$, given by ${ }^{(5)} G_{A B}={ }^{(5)} R_{A B}-\frac{1}{2} \gamma_{A B}{ }^{(5)} R$.

Variation of the action given in Eq. (1) w.r.t. $\phi$ results in

$\frac{2 \omega}{\phi}{ }^{(5)} \square \phi-\frac{\omega}{\phi^{2}} \nabla^{C} \phi \nabla_{C} \phi+{ }^{(5)} R-2 V^{\prime}(\phi)=0$,

where the prime ( $/$ ) denotes derivative with respect to $\phi$. Taking the trace of Eq. (2) we find

${ }^{(5)} R=\frac{\omega}{\phi^{2}} \nabla^{C} \phi \nabla_{C} \phi+\frac{8}{3} \frac{{ }^{(5)} \square \phi}{\phi}+\frac{10}{3} \frac{V(\phi)}{\phi}$,

which, when substituted in (3) yields

${ }^{(5)} \square \phi=-\frac{5 V(\phi)}{3 \omega+4}+\frac{3 V^{\prime}(\phi)}{3 \omega+4}$.

We shall show next how Eqs. (2) and (5) are reduced to $d=4$ in a particular cosmological setting, giving as a result the usual BD theory with the addition of an extra scalar field, whose dynamics and coupling to $\phi$ are determined by the reduction. $^{4}$

In the coordinate chart $\left\{y^{A}\right\}=\left\{x^{\mu}, z\right\}$ we consider the 5D line element

$\mathrm{d} s_{5}^{2}=\gamma_{A B} \mathrm{~d} y^{A} \mathrm{~d} y^{B}=\mathrm{d} t^{2}-a^{2}(t)\left(\mathrm{d} r^{2}+r^{2} \mathrm{~d} \Omega^{2}\right)-\xi^{2}(t) \mathrm{d} z^{2}$,

where $t$ is the time, $(r, \theta, \phi)$ are spherical coordinates on the hypersurfaces $t=$ constant, $z=$ constant, $z$ is the coordinate along the extra dimension, which we assume to be spacelike, and $\xi(t)$ is the metric coefficient associated to the extra dimension. The metric describing the standard cosmological model in $d=4$ is recovered by restricting this line element to a hypersurface $\Sigma_{0}$ defined by $z=z_{0}=$ constant.

In order to obtain the effective field equations in $d=$ 4 from the dimensional reduction of Eqs. (2) and (5), the following expressions were employed:

$\nabla_{\mu} \nabla_{\nu} \phi=\mathcal{D}_{\mu} \mathcal{D}_{\nu} \phi$

$\nabla_{z} \nabla_{z} \phi=-\xi\left(\mathcal{D}_{\alpha} \xi\right)\left(\mathcal{D}^{\alpha} \phi\right)$,

${ }^{(5)} \square \phi=\square \phi+\frac{\left(\mathcal{D}_{\alpha} \xi\right)\left(\mathcal{D}^{\alpha} \phi\right)}{\xi}$,

${ }^{(5)} R_{\mu \nu}=R_{\mu \nu}-\frac{\mathcal{D}_{\mu} \mathcal{D}_{\nu} \xi}{\xi}$,

${ }^{(5)} R_{z z}=\xi \square \xi$,

where $\mathcal{D}_{\alpha}$ denotes the 4D covariant derivative and $\square=$ $\mathcal{D}^{\alpha} \mathcal{D}_{\alpha}$. A long but straightforward calculation using all these expressions leads to the equations of the effective theory in $d=4$. The equation for the BD field that follows from Eq. (5) is

$\ddot{\phi}+3 H \dot{\phi}+\frac{\dot{\xi}}{\xi} \dot{\phi}=-\frac{5 V(\phi)}{3 \omega+4}+\frac{3 V^{\prime}(\phi)}{3 \omega+4}$.

From Eq. (2), with $A=B=0$, it follows that

$3 H^{2}+3 H \frac{\dot{\xi}}{\xi}=\frac{\omega}{2}\left(\frac{\dot{\phi}}{\phi}\right)^{2}-3 H \frac{\dot{\phi}}{\phi}-\frac{\dot{\xi}}{\xi} \frac{\dot{\phi}}{\phi}-\frac{V(\phi)}{\phi}$.

The spatial components of Eq. (2), corresponding to $A=i$ and $B=j$, can be written as

$$
\begin{aligned}
2 \dot{H} & +3 H^{2}+\frac{\ddot{\xi}}{\xi}+2 H \frac{\dot{\xi}}{\xi} \\
& =-\frac{\omega}{2}\left(\frac{\dot{\phi}}{\phi}\right)^{2}+H \frac{\dot{\phi}}{\phi}-\frac{V(\phi)(3 \omega-1)+3 \phi V^{\prime}(\phi)}{\phi(3 \omega+4)} .
\end{aligned}
$$

\footnotetext{
${ }^{4}$ For a generalization of this procedure to an arbitrary number of dimensions see [24].
} 
Table 1 Critical points of the system given by Eqs. (14)-(15) with $\dot{H}=0$. As explained in the text, only $P_{2 \pm}, P_{5 \pm}$, and $P_{6}$ will be considered in the subsequent analysis. The parameter $\beta$ is given by $\beta=\frac{1}{\Gamma-1}$

\begin{tabular}{|c|c|c|c|c|c|}
\hline Critical point & $\lambda$ & $x$ & $z$ & $y^{2}$ & Restriction on $\omega$ \\
\hline$P_{1}$ & $-\frac{5}{3}$ & 0 & 1 & -2 & - \\
\hline$P_{2 \pm}$ & 0 & $\frac{-3 \pm \sqrt{-15-12 \omega}}{\omega+2}$ & $-x-3$ & 0 & $\omega \leq-\frac{15}{12}$ \\
\hline$P_{3}$ & 0 & -4 & 1 & 0 & $\omega=-\frac{5}{4}$ \\
\hline$P_{4}$ & 0 & $-\frac{8}{3}$ & $-\frac{1}{3}$ & 0 & $\omega=-\frac{23}{16}$. \\
\hline$P_{5 \pm}$ & $\beta$ & $\frac{-3 \pm \sqrt{-15-12 \omega}}{\omega+2}$ & $\frac{-6 x(\omega+1)-9 \omega-6}{(3+x)(\omega+2)}$ & 0 & $\omega \leq-\frac{15}{12}$ \\
\hline$P_{6}$ & -1 & $\frac{1}{\omega+1}$ & 1 & $-\frac{1}{6} \frac{12 \omega^{2}+31 \omega+20}{(\omega+1)^{2}}$ & $-1.33 \leq \omega \leq-1.25$ \\
\hline
\end{tabular}

Finally, setting with $A=B=z$ in Eq. (2), we obtain

$3 \dot{H}+6 H^{2}=-\frac{\omega}{2}\left(\frac{\dot{\phi}}{\phi}\right)^{2}+\frac{\dot{\phi}}{\phi} \frac{\dot{\xi}}{\xi}-\frac{V(\phi)(3 \omega-1)+3 \phi V^{\prime}(\phi)}{\phi(3 \omega+4)}$.

These equations reduce to those presented in [11], when the vacuum and homogeneous case is considered in the latter. We shall show next that Eqs. (8)-(11) can be written as an autonomous 3-dimensional dynamical system.

\section{Dynamical system}

Techniques from dynamical systems theory have been successfully applied to cosmology, see for instance [25-28] for some recent examples. In terms of the variables (see for instance [29])

$$
\begin{aligned}
& x=\frac{\dot{\phi}}{H \phi}, \\
& y=\frac{1}{H} \sqrt{\frac{V(\phi)}{3 \phi}}, \\
& z=\frac{\dot{\xi}}{H \xi}, \\
& \lambda=-\phi \frac{V^{\prime}(\phi)}{V(\phi)} .
\end{aligned}
$$

Equation (9) is written as

$$
y^{2}=-1+\frac{1}{6} \omega x^{2}-\frac{1}{3} x z-z-x
$$

and acts as a constraint. From Eq. (11) it follows that

$$
\frac{\dot{H}}{H^{2}}=2 x+2 z-\frac{1}{2} \omega x^{2}+x z+\frac{3 y^{2}}{3 \omega+4}(\omega+\lambda+3) .
$$

The actual dynamical system follows from Eqs. (8)-(10), and it is given by

$$
\begin{aligned}
\frac{\mathrm{d} x}{\mathrm{~d} \tau}= & -x \frac{\dot{H}}{H^{2}}-x^{2}-3 x-x z-\frac{3(5+3 \lambda)}{3 \omega+4} y^{2}, \\
\frac{\mathrm{d} z}{\mathrm{~d} \tau}= & -(z+2) \frac{\dot{H}}{H^{2}}-z^{2}+4 x+z-\omega x^{2}+x z \\
& +\frac{3(5+3 \lambda)}{3 \omega+4} y^{2}, \\
\frac{\mathrm{d} \lambda}{\mathrm{d} \tau}= & x \lambda[1-\lambda(\Gamma-1)],
\end{aligned}
$$

where $\frac{\mathrm{d}}{\mathrm{d} \tau}=\frac{\mathrm{d}}{\mathrm{d} \ln a}$ and $\Gamma=\frac{V^{\prime \prime}(\phi) V(\phi)}{V^{\prime}(\phi)^{2}}$ is assumed to be a function of $\lambda$.

Table 1 shows the critical points of the system given by Eqs. (14)-(15), under the assumption that $\dot{H}=0$, which corresponds to a de Sitter expansion compatible with the latest observations, as mentioned in Sect. 1. We shall discard the critical point $P_{1}$, since it leads to $y^{2}<0$. Points $P_{3}$ and $P_{4}$ shall also be discarded because each of them is associated to a single value of $\omega$. Hence we shall focus the analysis on $P_{2 \pm}, P_{5 \pm}$, and $P_{6}$.

We shall study next the dynamical system given above by applying standard techniques, which include the introduction of new variables centered at the critical point, and the linearization of the system. From the linearization it is possible to calculate the Hubble parameter as a sum of terms each having a power of the expansion factor, where each power is given by an eigenvalue of the linearization matrix at the given critical point (for details, see [30] and the references therein). Hence we shall begin with the analysis of the behavior of the eigenvalues of the linearization matrix with $\omega$. The aim will be to obtain ranges for $\omega$ such that a given critical point is a stable node (for which all the eigenvalues must be real and negative), or a stable focus (characterized by one real and negative eigenvalue, and two complex eigenvalues with negative real parts).

The linearization matrix of the system in Eqs. (14)-(15) at a given critical point is given by 
$A=\left[\begin{array}{ccc}\left.A_{11}\right|_{c} & -\frac{\left(\lambda_{c}-2 \omega-1\right) x_{c}{ }^{2}+2\left(3 \lambda_{c}-3 \omega+1\right) x_{c}+3\left(3 \lambda_{c}+5\right)}{(3 \omega+4)} & -\frac{\omega x_{c}{ }^{3}+\left(z_{c}-3(\omega-2)\right) x_{c}{ }^{2}+6\left(z_{c}+2\right) x_{c}+9\left(z_{c}+1\right)}{2(3 \omega+4)} \\ \left.A_{21}\right|_{c} & -\frac{\left(\lambda_{c}-2 \omega-1\right)\left(\omega x_{c}{ }^{2}-2\left(z_{c}-1\right) x_{c}-12 z_{c}\right)}{2(3 \omega+4)} & -\frac{\left(1-z_{c}\right) \omega x_{c}{ }^{2}+2\left(z_{c}-1\right)\left(z_{c}+3\right) x_{c}+6\left(z_{c}{ }^{2}-1\right)}{2(3 \omega+4)} \\ \lambda_{c}\left(1-\lambda_{c}\left(\Gamma\left(\lambda_{c}\right)-1\right)\right) & 0 & -\left.\frac{\mathrm{d} \Gamma(\lambda)}{\mathrm{d} \lambda}\right|_{\lambda_{c}} \lambda_{c}{ }^{2} x_{c}-2 \Gamma\left(\lambda_{c}\right) \lambda_{c} x_{c}+2 x_{c} \lambda_{c}+x_{c}\end{array}\right]$

where

$$
\begin{aligned}
\left.A_{11}\right|_{c}= & -\frac{1}{2(3 \omega+4)}\left[4\left(3 \omega-3 \lambda_{c}-1\right) z_{c}\right. \\
& +3\left(\lambda_{c}-2 \omega-1\right) \omega x_{c}{ }^{2}+\left(6(\omega-2) \lambda_{c}+34 \omega+12\right. \\
& \left.\left.+\left(-4 \lambda_{c}+8 \omega+4\right) z_{c}\right) x_{c}\right], \\
\left.A_{21}\right|_{c}= & -\frac{2}{3 \omega+4}\left[\left(\lambda_{c}-2 \omega-1\right)\left(z_{c}-1\right) \omega x_{c}+\right] \\
& \times\left(-\lambda_{c}+\omega+1\right)\left(z_{c}^{2}+2 z_{c}-3\right) .
\end{aligned}
$$

We shall analyze next the behavior with $\omega$ of the eigenvalues of this matrix at each critical point.

\section{$3.1 \mathbf{P}_{2 \pm}$}

Since $\lambda=0$ for these critical points, it follows from the expression of the matrix $A$, given in Eq. (16), that the eigenvalues do not depend on the explicit expression of $\Gamma$. Hence, the results that follow will be valid for $\phi=\phi_{c}=0$ and $\left.\left(V^{\prime} / V\right)\right|_{\phi_{c}}$ finite, or $V^{\prime}\left(\phi_{c}\right)=0$.

\subsection{1 $P_{2+}$}

The eigenvalues of the matrix $A$ for this critical point are given by

$$
\begin{aligned}
a_{1}(\omega)= & -\frac{1}{2(3 \omega+4)(\omega+2)^{2}}\left[-30 \omega-24-9 \omega^{2}\right. \\
& +\sqrt{-15-12 \omega}\left(10 \omega+3 \omega^{2}+8\right) \\
& -\left(-2304 \omega^{6}+4116 \omega^{5}\right. \\
& +48906 \omega^{4}+105600 \omega^{3}+99600 \omega^{2}+43776 \omega \\
& +7296+\sqrt{-15-12 \omega}\left(-2976 \omega^{5}-10134 \omega^{4}\right. \\
& \left.\left.\left.-11424 \omega^{3}-3888 \omega^{2}+768 \omega+384\right)\right)^{1 / 2}\right],
\end{aligned}
$$

$a_{2}(\omega)=\frac{3-\sqrt{-15-12 \omega}}{\omega+2}-a_{1}(\omega)$,

$a_{3}(\omega)=\frac{-3+\sqrt{-15-12 \omega}}{\omega+2}$.

Figure 1 shows the behavior with $\omega$ of the real part of each eigenvalue associated to $P_{2+}$. The plots show that there are no values of $\omega$ such that the real parts of the three eigenvalues are real and negative. Consequently, $P_{2+}$ cannot be a stable point, and the behavior of the system close to $P_{2+}$ cannot approach the one currently displayed by the $\Lambda \mathrm{CDM}$ model.

\subsection{2 $P_{2-}$}

The eigenvalues in this case are given by the following expressions:

$$
\begin{aligned}
a_{1}(\omega)= & \frac{1}{2(3 \omega+4)(\omega+2)^{2}}[\sqrt{6}(1216+7296 \omega \\
& +16600 \omega^{2}+17600 \omega^{3}+8151 \omega^{4}+686 \omega^{5} \\
& -384 \omega^{6}+\sqrt{-15-12 \omega}\left(-64-128 \omega+648 \omega^{2}\right. \\
& \left.\left.+1904 \omega^{3}+1689 \omega^{4}+496 \omega^{5}\right)\right)^{1 / 2}+9 \omega^{2}+30 \omega \\
& \left.+24+\left(3 \omega^{2}+10 \omega+8\right) \sqrt{-15-12 \omega}\right], \\
a_{2}(\omega)= & \frac{3+\sqrt{-15-12 \omega}}{\omega+2}-a_{1}(\omega), \\
a_{3}(\omega)= & -\frac{3+\sqrt{-15-12 \omega}}{\omega+2} .
\end{aligned}
$$

Figure 2 shows the plots of the real part of each eigenvalue associated to $P_{2-}$. The plots show that there is no interval of

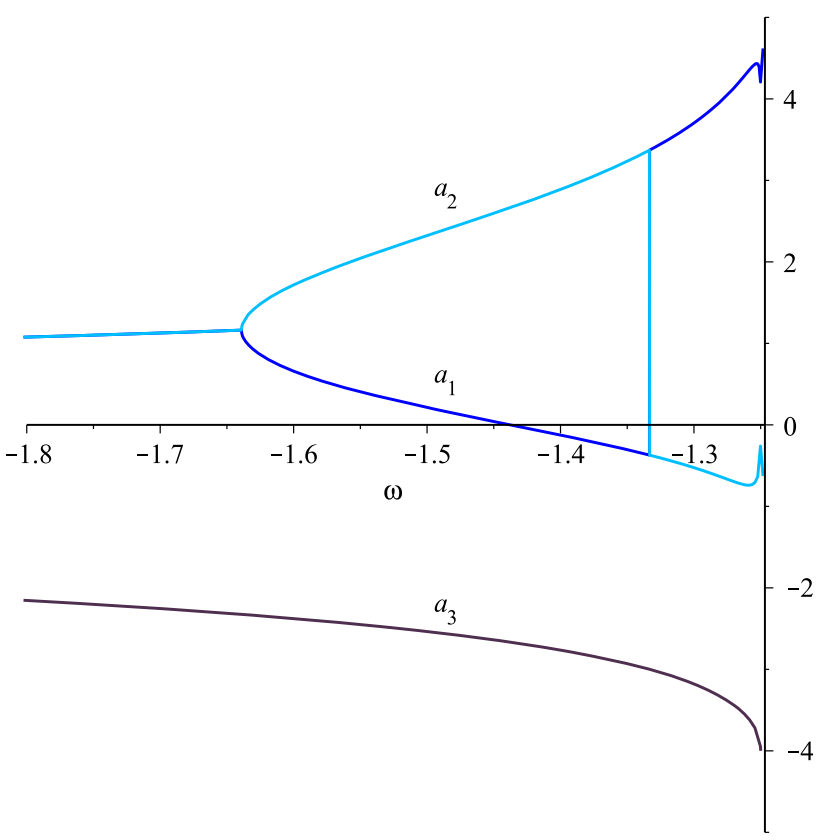

Fig. 1 Real parts of the eigenvalues corresponding to $P_{2+}$. The curves corresponding to $a_{1}$ and $a_{2}$ are superposed to the left of approx. $\omega=$ -1.64 , and they show a discontinuity at $\omega=-4 / 3$ 


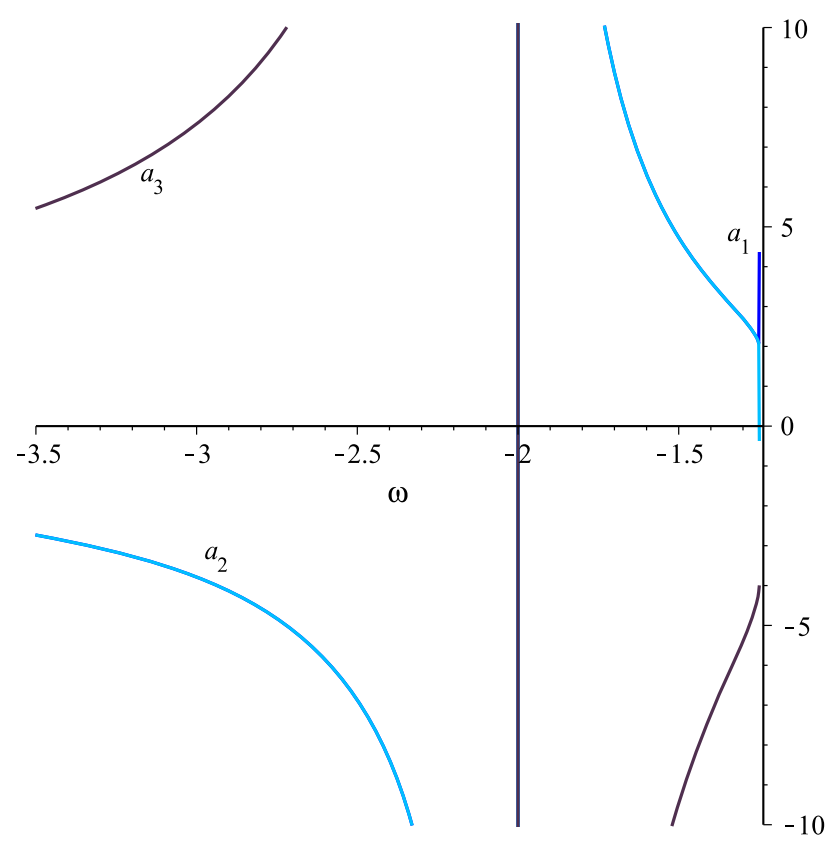

Fig. 2 Real parts of the eigenvalues corresponding to $P_{2-}$. The curves corresponding to $a_{1}$ and $a_{2}$ are not superposed only near $\omega=-1.25$, and they show a divergence for $\omega=-2$

values of $\omega$ such that the real parts of the three eigenvalues are negative.

\section{$3.2 \mathbf{P}_{5 \pm}$}

The critical points $P_{5 \pm}$ depend on the expression for the potential through the condition $\lambda=\beta$ (see Table 1). Since the eigenvalues for arbitrary values of $\omega$ and $\beta$ are given by

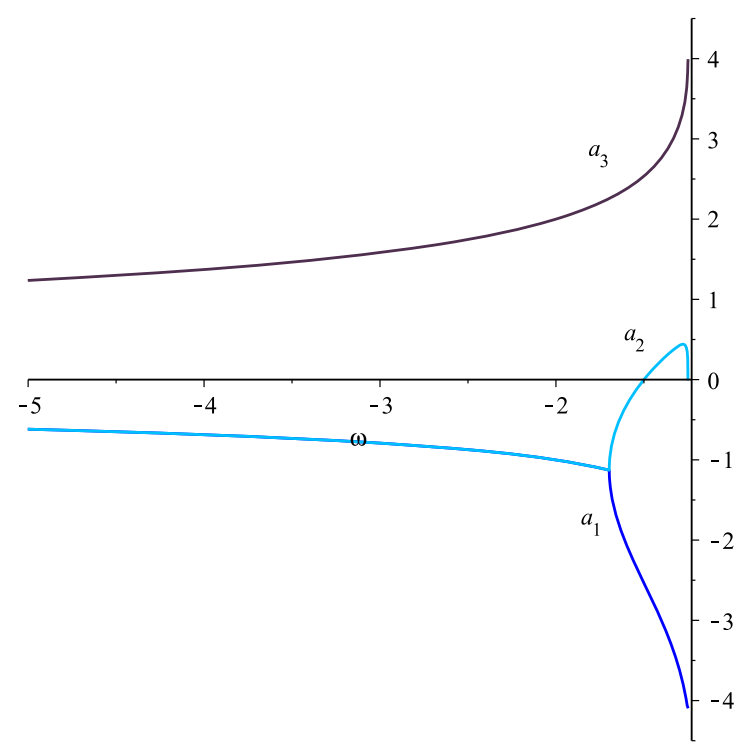

long algebraic expressions, we restrict here to the potential $V(\phi)=V_{0} \phi^{n}$, such that $\beta=\lambda=-n$ for every value of $V_{0}$ and $n$. This choice is justified by the fact that several effective quantum field theories can be related to his kind of self-interacting potential [31]. In particular, we shall examine the cases $n=2$ and $n=4$, frequently considered in cosmological scenarios (see for instance $[21,32,33]$ ).

\subsection{1 $P_{5+}$}

The real parts of the eigenvalues corresponding to the critical point $P_{5+}$ are plotted in Fig. 3 for $n=2$ and $n=4$. None of the cases is associated to a stable critical point with $\dot{H}=0$.

\subsection{2 $P_{5-}$}

The eigenvalues are plotted in Fig. 4 for $n=2$ and $n=4$, and they fail to comply with the condition that their real parts be negative.

\section{$3.3 \mathbf{P}_{\mathbf{6}}$}

The expressions for the eigenvalues in this case are the following:

$$
\begin{gathered}
a_{1}(\omega)=-\frac{\left.\frac{\mathrm{d} \Gamma(\lambda)}{\mathrm{d} \lambda}\right|_{\lambda_{c}}+1}{\omega-1}, \\
a_{2}(\omega)=a_{3}(\omega)=-\frac{4 \omega+5}{\omega-1},
\end{gathered}
$$

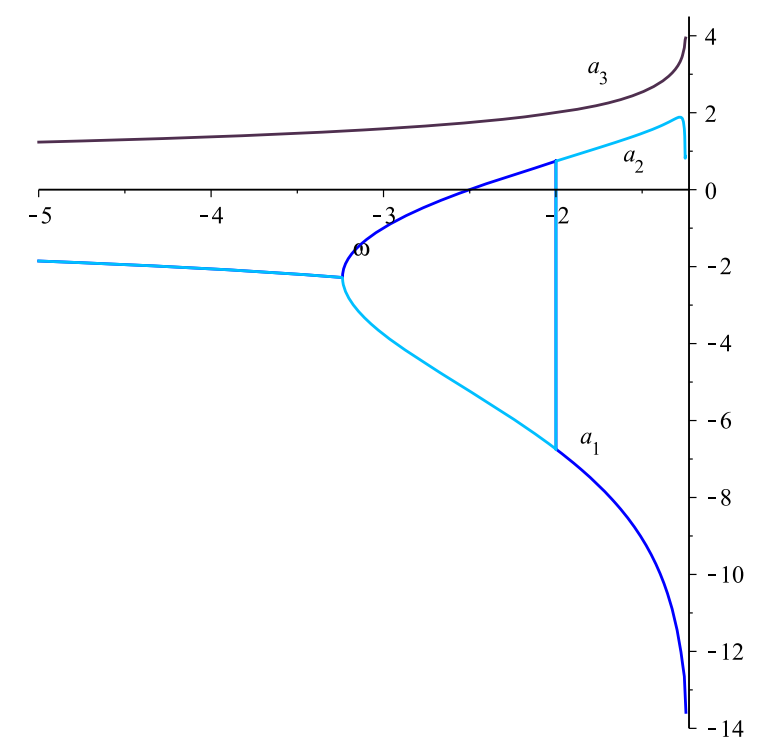

Fig. 3 Real parts of the eigenvalues corresponding to $P_{5+}$, for $n=2$ (left), and $n=4$ (right). The latter shows a discontinuity for $\omega=-2$. The plots for $a_{1}$ and $a_{2}$ are superposed for $\omega \lesssim-1.7$ (left) and $\omega \lesssim-3.2$ (right) 

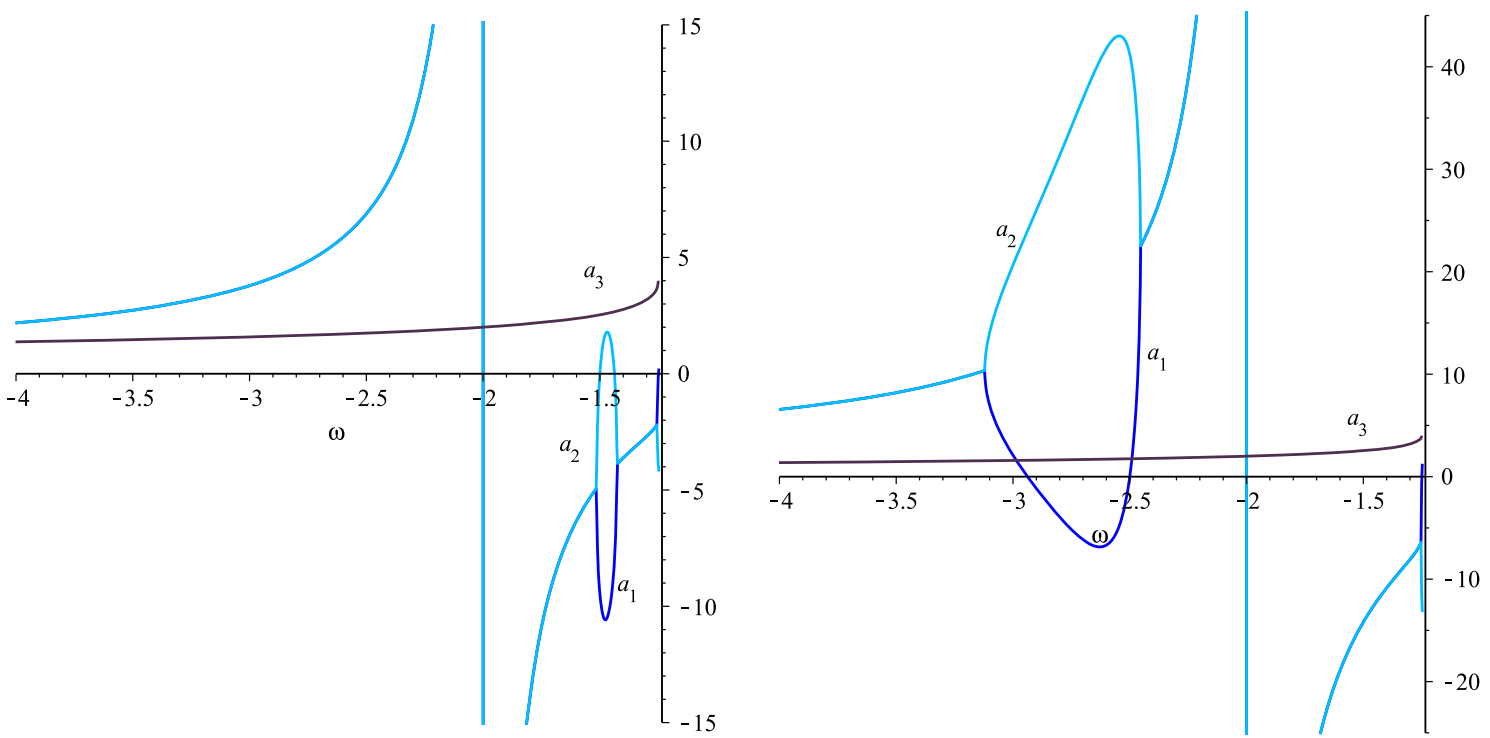

Fig. 4 Real parts of the eigenvalues corresponding to $P_{5-}$, with $n=2$ (left) and $n=4$ (right). $a_{1}$ and $a_{2}$ are singular at $\omega=-2$

with $\Gamma(-1)=0 .^{5}$ The eigenvalues are shown in Fig. 5 for $\left.\frac{\mathrm{d} \Gamma(\lambda)}{\mathrm{d} \lambda}\right|_{\lambda_{c}}=-1.8$. We see that, in spite of the fact that the real parts of the three eigenvalues are negative, the eigenvalue $a_{1}$ could be associated to non-relativistic matter ( i.e. is such that $\operatorname{Re}\left(a_{1}\right)=-3$ ) only for a unique value of $\omega$. Note that, although this conclusion follows from a particular value of $\left.\frac{\mathrm{d} \Gamma(\lambda)}{\mathrm{d} \lambda}\right|_{\lambda_{c}}$, the same will happen for any other value of the derivative compatible with the restrictions, due to the specific form of the dependence of $a_{1}$ with the derivative. Hence, $P_{6}$ should also be discarded.

\section{Discussion}

We have examined whether a 4-dimensional universe in accelerated expansion and containing non-relativistic matter can be obtained by dimensional reduction of a self-interacting BD theory defined in $d=5$. The study required rewriting the equations of the system as an autonomous 3-dimensional dynamical system. The analysis of the eigenvalues of the linearized system shows that it has no stable equilibrium points subject to the condition $\dot{H}=0$, except for the critical point $P_{6}$, which is a stable critical point but can describe non-relativistic matter only for a unique value of $\omega$ (given a value of $\left.\frac{\mathrm{d} \Gamma(\lambda)}{\mathrm{d} \lambda}\right|_{\lambda_{c}}$ compatible with the restrictions). Hence, the model cannot mimic the $\Lambda \mathrm{CDM}$ dynamics. This conclusion was obtained in full generality for $P_{2 \pm}$ and $P_{6}$, and for

5 The explicit form of the potential can in principle be obtained from any function $\Gamma(\lambda)$ such that $\Gamma(-1)=0$, and $\left.\frac{\mathrm{d} \Gamma(\lambda)}{\mathrm{d} \lambda}\right|_{\lambda=-1}=$ constant, and from the definition of $\lambda$.

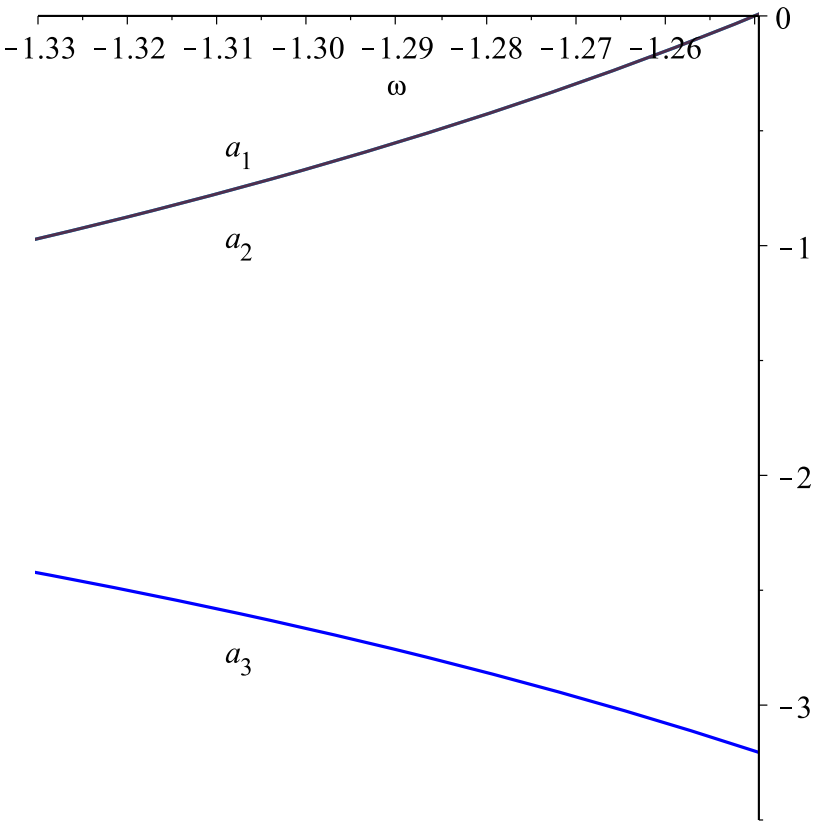

Fig. 5 Plot of the real parts of the eigenvalues corresponding to $P_{6}$, for $\left.\frac{\mathrm{d} \Gamma(\lambda)}{\mathrm{d} \lambda}\right|_{\lambda_{c}}=-1.8$

$V(\phi)=V_{0} \phi^{n}$ and $n=2,4$ in the case of $P_{5 \pm}$. The failure of the model presented here in describing both the accelerated expansion and the matter content of the 4-dimensional universe should perhaps be taken as an indication that more complex models are needed, such as those presented in [11], where the metric coefficient of the extra dimension is a function of both time and the extra coordinate. We hope to come back to these ideas in a future publication. 
Acknowledgements This work was supported by PROSNI 2015-2016, PROFOCIE 2015-2016, P3E 235947 PROMOFID 2017, and Centro Universitario de Ciencias Exactas e Ingenierias of Universidad de Guadalajara

Open Access This article is distributed under the terms of the Creative Commons Attribution 4.0 International License (http://creativecomm ons.org/licenses/by/4.0/), which permits unrestricted use, distribution, and reproduction in any medium, provided you give appropriate credit to the original author(s) and the source, provide a link to the Creative Commons license, and indicate if changes were made.

Funded by SCOAP ${ }^{3}$.

\section{References}

1. Alves Joao, Combes Françoise, Ferrara Andrea, Forveille Thierry, Shore Steve, Planck 2015 results. A\&A 594, E1 (2016)

2. Joshua Frieman, Michael Turner, Dragan Huterer, Dark energy and the accelerating universe. Ann. Rev. Astron. Astrophys. 46, 385432 (2008)

3. Sean M. Carroll. Why is the universe accelerating? eConf, C0307282:TTH09, (2003). [AIP Conf. Proc.743,16(2005)]

4. Li Miao, Li Xiao-Dong, Wang Shuang, Wang Yi, Dark energy. Communications in theoretical physics 56(3), 525 (2011)

5. Timothy Clifton, Pedro G. Ferreira, Antonio Padilla, Constantinos Skordis, Modified gravity and cosmology. Phys. Rept. 513, 1-189 (2012)

6. K. Bolejko, M. Korzyńki. Inhomogeneous cosmology and backreaction: current status and future prospects. (2016)

7. P. S. Wesson. Five-dimensional physics: Classical and quantum consequences of Kaluza-Klein cosmology (2006)

8. S. Wesson Paul, J. de Ponce Leon, Kaluza-klein equations, einstein's equations, and an effective energy-momentum tensor. J. Math. Phys. 33(11), 3883-3887 (1992)

9. J. Ponce de Leon, Late time cosmic acceleration from vacuum Brans-Dicke theory in 5D. Class. Quant. Grav. 27, 095002 (2010)

10. L. M. Reyes, J. E. Madriz Aguilar. Embedding general relativity with varying cosmological constant term in five-dimensional Brans-Dicke theory of gravity in vacuum. ArXiv e-prints, February 2009

11. J. Ponce de Leon, Brans-Dicke cosmology in 4D from scalarvacuum in 5D. JCAP 1003, 030 (2010)

12. Amir F. Bahrehbakhsh, Mehrdad Farhoudi, Hossein Shojaie, FRW cosmology from five dimensional vacuum Brans-Dicke theory. Gen. Rel. Grav. 43, 847-869 (2011)

13. S.M.M. Rasouli, M. Farhoudi, H.R. Sepangi, An anisotropic cosmological model in a modified Brans-Dicke theory. Class. Quantum Grav. 28(15), 155004 (2011)

14. S.M.M. Rasouli, Paulo Vargas Moniz, Exact cosmological solutions in modified Brans-Dicke theory. Class. Quant. Grav. 33(3), 035006 (2016)
15. Li-e Qiang, Yong-ge Ma, Mu-xin Han, Yu. Dan, 5-dimensional Brans-Dicke theory and cosmic acceleration. Phys. Rev. D 71, 061501 (2005)

16. Amir F. Bahrehbakhsh, Mehrdad Farhoudi, Hajar Vakili, Dark energy from fifth dimensional Brans-Dicke theory. Int. J. Mod. Phys. D 22, 1350070 (2013)

17. Ahmad Borzou, Hamid Reza Sepangi, Shahab Shahidi, Razieh Yousefi, Brane f(R) gravity. Europhys. Lett. 88(2), 29001 (2009)

18. Antonio Troisi, Higher-order gravity in higher dimensions: Geometrical origins of four-dimensional cosmology? Eur. Phys. J. C 77(3), 171 (2017)

19. H.R.S. Pedro, Moraes. Cosmological solutions from induced matter model applied to 5D $f(R, T)$ gravity and the shrinking of the extra coordinate. Eur. Phys. J. C75(4), 168 (2015)

20. C. Santos, R. Gregory, Cosmology in Brans-Dicke theory with a scalar potential. Ann. Phys. 258, 111-134 (1997)

21. S. Sen, T.R. Seshadri, Self interacting Brans-Dicke cosmology and quintessence. Int. J. Mod. Phys. D 12, 445-460 (2003)

22. W. Chakraborty, U. Debnath, Role of Brans-Dicke theory with or without self-interacting potential in cosmic acceleration. Int. J. Theoret. Phys. 48, 232-247 (2009)

23. L. Perivolaropoulos, Equation of state of the oscillating brans-dicke scalar and extra dimensions. Phys. Rev. D 67, 123516 (2003)

24. S.M.M. Rasouli, Mehrdad Farhoudi, Paulo Vargas Moniz, Modified Brans-Dicke theory in arbitrary dimensions. Class. Quantum Grav. 31, 115002 (2014)

25. G. Acquaviva, A. Beesham, Nonlinear bulk viscosity and the stability of accelerated expansion in frw spacetime. Phys. Rev. D 90, 023503 (2014)

26. Athira Sasidharan, Titus K. Mathew, Phase space analysis of bulk viscous matter dominated universe. J. High Energy Phys. 2016(6), 138 (2016)

27. M. Sharif, Saadia Mumtaz, Stability of the accelerated expansion in nonlinear electrodynamics. Eur. Phys. J. C 77(2), 136 (2017)

28. M. Shahalam, S.D. Pathak, Shiyuan Li, R. Myrzakulov, Anzhong Wang, Dynamics of coupled phantom and tachyon fields. Eur. Phys. J. C 77(10), 686 (2017)

29. Orest Hrycyna, Marek Szydlowski, Brans-Dicke theory and the emergence of $\Lambda$ CDM model. Phys. Rev. D 88(6), 064018 (2013)

30. Orest Hrycyna, Marek Szydlowski, Uniting cosmological epochs through the twister solution in cosmology with non-minimal coupling. JCAP 1012, 016 (2010)

31. Y. Fujii, K.-I. Maeda. The scalar-tensor theory of gravitation. (2003)

32. B. Boisseau, H. Giacomini, D. Polarski, Bouncing universes in scalar-tensor gravity around conformal invariance. J. Cosmol. Astropart. Phys. 5, 048 (2016)

33. S. Carloni, J.A. Leach, S. Capozziello, P.K.S. Dunsby, Cosmological dynamics of scalar tensor gravity. Class. Quantum Grav. 25(3), 035008 (2008) 